\title{
THE RESTORATION OF NORMAL BREATHING IN ASTHMATIC CHILDREN
}

\author{
BY \\ ARNOLD A. NOVE \\ From the Asthma Clinic of the Royal Chest Hospital, London
}

(RECEIVED FOR PUblication JANUARY 8, 1952)

This preliminary report records a clinical study of the relation between the bony framework of the skull and respiratory function conducted at the Royal Chest Hospital over a period of five years. The purpose is to present a new angle of approach to the problem of asthma in childhood.

Most of the commonly accepted methods of treatment aim at the correction of an allergic diathesis, organic disease in the upper respiratory tract, infection or a psychological state. These methods may be successful, but it is well known that the asthma may recur. A successful result of the course of treatment outlined in this paper causes a restoration to normal of a previously abnormal upper respiratory tract by altering anatomical relations. This accomplished, there would seem to be a much greater chance that the improvement produced in the mechanics of breathing will prove to be permanent.

In a high percentage of asthmatic children there is a malformation of the bony framework of the face which has proved capable of being corrected without surgical intervention, and therefore this investigation was approached from the aspect of considering to what extent the various types of facial and other skeletal deformity characteristic of the asthmatic may be an aetiological factor in the disease and its assóciated abnormal posture.

Normal posture is a physiological expression of a balanced mechanism manifested in rhythmic movement and static poise, whether it concerns the entire organism or a particular component. The postural adjustments characteristic of the asthmatic during phases of activity and rest represent an effort at accommodation towards adequate respiratory function by an organism in a state of imbalance. Structure and function being interdependent, it is reasonable to postulate that an inherent structural fault, which might impose a need for postural accommodation, could be an important feature in the production of the asthmatic habitus. It is also probable that the relatively greater expenditure of neuromuscular energy required by an ill-balanced organism to maintain asymmetric postural positions, even during rest, tends not only to maintain but to accentuate a vicious circle.

The writer, while engaged upon another clinical investigation, observed that children with underdeveloped and malrelated jaws have not only facial asymmetry, but also tend to develop a stooping posture, winged scapulae and a liability to postnasal catarrh and other respiratory symptoms (Nove, 1945).

It became apparent from a close study of the mechanism of mastication, deglutition and respiration in a number of children presenting a variety of the malformations that the action of the muscles of the face and neck lacked the synergy of action to be seen in those whose skeletal and muscular formation were properly balanced. It was also found that this lack of harmony could be reduced by employing orthopaedic methods.

The mechanism employed was introduced originally as an orthodontic appliance by Robin (1929) and further developed by Andresen (1938). Its application and construction, however, were adapted by the writer to the orthopaedic requirements of correcting a faulty integration of the structures which comprise the cervico-facial component (Nove, 1946a). The object is to correct the abnormality of the bony framework of the face and thus to improve the mechanism of respiration. In time it is found that a more normal airway is the result. For this purpose the maxilla is regarded as the proximal end and the mandible the distal end of the component.

It is expedient to begin the first stage of the orthopaedic procedure extra-orally. Plaster casts are obtained in the ordinary way, care being taken to ensure that they are sharply defined and extend beyond the junction of the hard and soft palate and tuberosities in the upper jaw, and that the lower cast is deep enough to include the sites of the 
mylohyoid ridges and geniohyoid tubercle. These anatomical constants are readily discernible on the casts and are important landmarks which guide the clinician in plotting out a mandibular base line from which to determine the correct vertical and antero-posterior relationship of the jaws (Nove, 1946b). This aspect of the procedure has been discussed by Campbell (1950).

A roll of soft wax is placed between the ' occlusal' surfaces of the teeth of the upper and lower models which are then manoeuvred until a correct inter-maxillary relationship appears to have been found. This provisional wax 'block' is then placed on the occlusal surface of the lower jaw, which is guided to the upper jaw so that the teeth bite into the corresponding facets made in the wax, and the patient is instructed to close the mouth; an intra-oral splint is now fashioned in a plastic material. The details of the mechanical procedure are outside the scope of this report. The purpose of the splint is to retain the new inter-maxillary relationship intermittently, specially during the night.

When a splint made to a correctly plotted orientation is placed in the mouth, certain characteristic behaviour patterns of the structures concerned occur as the splint engages both jaws. The orbicularis oris closes, initiating an act of swallowing which, in the normal, has a functional pattern of its own (Nove, 1948), and is identified by a synchronized movement in the angle of the jaw and neck as the thyroid prominence is seen to trace the excursion made by the tongue and associated structures in an upward and slightly forward direction, registering a vigorous synergic contraction of the facial and neck muscles, ending in relaxation.

The synergy of muscular action exhibited during this process and the fact that it is followed by an expression of greater repose, suggestive of a state of physiological rest, indicate that the bony framework of the face has been adequately augmented and the requisite fulcrum for full muscular action provided, thereby reducing faulty integration in the internal and external structures of the face and neck. An alteration in cranio-cervical posture takes place and an appreciable improvement in nasal breathing and costo-diaphragmatic rhythm follows immediately. Sometimes the change is dramatic, but generally it fluctuates to some extent before normal breathing is restored.

Provided that the patient can co-operate fully and that the clinician is meticulously careful in maintaining the requisite structural balance, smooth respiratory excursion ensues, symptoms gradually disappear and, in due course, normal health is established. At the same time the interaction imposed by the newly introduced balanced muscular stresses has the corresponding effect of remoulding the bony framework, of the face, including the temporo-mandibular joints, in accordance with accepted principles of bone reconstruction known since the days of John Hunter and fully discussed by Brailsford (1945). Sicher (1947) treats of the special characteristic features concerning the growth and development of the bony elements of the structures under discussion.

The length of time required for reconstruction from a faulty structure of the face to one that is so balanced as to restore normal breathing varies with the age of the patient at the beginning of treatment and the degree of structural fault. If treatment begins at 3 years of age or earlier the minimum period required is, on an average, three years. Similarly, children with mixed dentition and adolescents require on an average between four and five years to restore the normal structure. That is to say, although improved respiratory function follows shortly after the patient begins to wear the splint, unaided normal breathing cannot be assured until the bony framework of the face has been adequately reshaped, establishing a harmonious interplay between the internal and external structures of the head and neck component. Only then can the splint be discarded.

In the course of the treatment frequent adjustments to the splint, as well as new orientations and new splints, have to be made to meet the constant changes of growth and development in order to maintain and eventually consolidate the improved structural balance and cranio-cervical and general posture.

To maintain this biological process constant supervision is required. The balance of this equilibrium is a very delicate one; for example, the cusp or incisal edge of an erupting permanent tooth impinging on the splint produces a ' high spot' (with or without pain) and can disturb the equilibrium sufficiently to reverse an otherwise favourable clinical course, for when the bony fulcrum is even slightly upset functional imbalance is reintroduced.

The facial skeleton, no less than other bones, provides a fulcrum for muscular contractions and support during physiological rest. If the fulcrum is out of true because of faulty skeletal formation, function is forced into a variety of patterns of adaptive forms (Nove, 1948). Intra-oral splints made to correct anatomical faults have, therefore, to be renewed every few months as well as frequently adjusted, in order to keep in step with the 
process of growth and development, as well as to maintain the required equilibrium for normal muscular activity, until the bony support has been helped by the interaction between structure and function sufficiently to reach that maximum reconstruction at which the correct functional interplay will maintain the required balance.

\section{Clinical Data}

This study concerns a group of 74 asthmatic children between the ages of 3 and 16. (The average age at the beginning of treatment was 9 years.) The cases were selected from patients attending the Clinic between December, 1943, and November, 1948, as suitable for treatment because most had proved resistant to the usual methods and thereby provided a form of control, and, in addition, their attendance had been fairly regular. In each case the presenting symptom was asthma.

In order not to invalidate the scientific value of the conclusions to be derived from the method of treatment described, patients (or parents) were also instructed to discontinue other remedies and to get in touch with the hospital or their doctor should they have an acute attack of asthma. Also, whenever possible, the family doctor was asked to refrain from prescribing and to render only such aid as an emergency might necessitate.

A full clinical and pathological examination was carried out in each case before the patient was accepted as being suitable for treatment. The general outline of the investigation followed that in routine use in the Asthma Clinic at the Royal Chest Hospital and described elsewhere by Maxwell (1936). Analysis of the factors present showed results which were comparable with those reported in other series.

Allergy. In every case the history, family history, and the results of skin tests were taken into consideration. An allergic factor was considered to be present in 44 of the cases.

The Upper Respiratory Tract. The nose and throat were examined by routine methods, including radiography in doubtful cases. Various abnormalities, such as 'collapsed' nares, faulty nasal airway, mouth breathing, infected sinuses, clinically infected tonsils, granular mucosa, or ear discharge, were reported in 49 cases.

The Lower Respiratory Tract. Bronchitis, emphysema and a mild degree of bronchiectasis were the commonest abnormalities discovered, and in addition, there was a considerable chest deformity in a number of patients. In- all, it was considered that the bronchopulmonary system showed evidence of organic change in 26 cases.
The Psychological Factor. This is the most difficult of all factors to assess in asthmatic children, for it is hard to know how much of the nervous symptoms is a part of the child's constitution and how much has been produced by previous attempts at medical or surgical treatment. In addition, it is not always possible to assess fully the patient's background, and yet it is well recognized that an unsatisfactory home environment is a very common cause of the persistence of symptoms. From information obtained from parents and other sources, there appeared to be a significant psychological abnormality in 30 of these cases.

During the first five years of this investigation the routine examination and clinical assessment were carried out by the writer jointly with Dr. Beryl Barsby, formerly Registrar at the Royal Chest Hospital. The final clinical assessment and classification of every case was carried out by Dr. James Maxwell.

\section{Results}

It is never easy to present the results of treatment in asthma, for the multiplicity of factors concerned make it difficult to form a balanced judgment on the effect of a particular remedy. The only possible criterion is the cessation of attacks of asthma, and, therefore, cases have been divided into three groups.

Group 1 (34 Cases). The patients in this group have all been completely free from asthma for at least one year and many for longer periods. They are all engaged in full-time school activities and they are capable of taking part in the usual sports and games without restriction.

Group 2 (20 Cases). In this group the result is not quite so satisfactory, although every child is leading a normal life. There is no significant interference with attendance at school, but there are occasional attacks of asthma and full physical exercise is not achieved.

Group 3 (20 Cases). No significant improvement is seen in this group.

There was a significantly constant rise in the curve of the vital capacity graph in most of the cases under treatment, and radiographic evidence of favourable bony changes in the thoracic cage, as well as in the facial bones, supporting the subjective evidence. A point of interest was that neither the allergic nor the psychological factor appeared to influence the clinical course of the series of cases under review.

A favourable prognosis seems to be dependent on (1) full co-operation on the part of the patient; (2) successful reduction of structural imbalance; (3) avoidance of expenditure of energy beyond the 
patient's physical capacity in the early phases of treatment. As a result of the feeling of relief experienced, the patient tends, understandably, to overstep the respiratory threshold reached. Failure to exercise restraint may in some cases interfere with a favourable clinical course; (4) an adequate length of time is required for treatment, because the structural changes which permanently establish normal respiratory function are inevitably gradual and individually variable.

\section{Summary and Conclusions}

A group of 74 children was studied in which the presenting symptom was asthma; all of them showed some form of faulty jaw formation. Symptoms did not appear to relate to any particular type of deformity, and the type generally referred to as adenoid facies appeared to respond to the treatment as readily as other types.

The clinical findings were made under conditions approaching control and suggest that the orthopaedic method evolved offers a means whereby normal respiratory function may be restored in asthmatic children whilst resolving at the same time a structural fault which may be the causative factor. The method is also of value in clinical research.

I am most grateful to Dr. James Maxwell for the opportunity to develop the investigation and for very generous guidance throughout; to Dr. Beryl Barsby, who meticulously compiled and collated much clinical data; and to Mr. Ian Robin for his evaluation of some of the cases.

REFERENCES

Andresen, V. Cited in Grude, R. (1938). Dent. Rec., 58, 529.

Brailsford, J. F. (1945). Brit. J. Surg., 32, 345.

Campbell, J. (1950). Dent. Rec., 70, 204.

Maxwell, J. (1936). Brit. med. J., 1, 874.

Nove, A. A. (1945). Proc. roy. Soc. Med., 38, 540.

- (1946a). Dent. Rec., 66, 25.

(1946b). Ibid., 66, 55 .

- (1948). Ibid., 68, 28.

Robin, P. (1929). Eumorphie. La Glossoptose. Paris.

Sicher, H. (1947). Amer. J. Orthodont., 33, 30. 